\title{
Salidroside inhibits the proliferation and migration of gastric cancer cells via suppression of Src-associated signaling pathway activation and heat shock protein 70 expression
}

\author{
ZHILIN QI $^{1,2}$, TUO TANG $^{2}$, LILI SHENG ${ }^{3}$, YUNFEI MA ${ }^{2}$, YINHUA LIU $^{4}$, \\ LIANG YAN $^{1,2}$, SHIMEI QI ${ }^{1,2}$, LIEFENG LING $^{1,2}$ and YAO ZHANG ${ }^{1,2}$ \\ ${ }^{1}$ Department of Biochemistry; ${ }^{2}$ Anhui Province Key Laboratory of Active Biological Macromolecules, \\ Wannan Medical College; Departments of ${ }^{3}$ Oncology and ${ }^{4}$ Pathology, Yijishan Hospital, \\ Wannan Medical College, Wuhu, Anhui 241002, P.R. China
}

Received October 25, 2017; Accepted April 9, 2018

DOI: $10.3892 / \mathrm{mmr} .2018 .8958$

\begin{abstract}
Salidroside, an active ingredient extracted from the Rhodiola rosea plant, has potential anti-tumor effects. However, the effects of salidroside on gastric cancer cell proliferation and migration remain unclear. In the present study, the inhibitory effects of salidroside on gastric cancer cell proliferation, migration and invasion and the molecular mechanisms underlying these effects were investigated. The human gastric cancer cell line, BGC-823, was treated with different concentrations of salidroside (200, 400 and $600 \mu \mathrm{g} / \mathrm{ml})$. Cell proliferation was determined with Cell Counting Kit- 8 and colony formation assays, and the migration and invasion of cells was detected by a wound healing and Transwell assay, respectively. Western blotting was performed to detect the levels of N-cadherin, E-cadherin and heat shock protein (HSP)70. In addition, the phosphorylation of proto-oncogene tyrosine-protein kinase $\mathrm{Src}(\mathrm{Src})$, protein kinase B (Akt), mitogen activated protein kinase 1 (ERK), signal transducer and activator of transcription (STAT) 3 and focal adhesion kinase 1 (FAK) was examined by western blotting. The levels of matrix metalloproteinase (MMP)-2 and MMP-9 were determined by enzyme-linked immunosorbent assay kits. Levels of reactive oxygen species (ROS) in cells were measured by a fluorescence plate reader with
\end{abstract}

Correspondence to: Dr Zhilin Qi or Dr Yao Zhang, Department of Biochemistry, Wannan Medical College, 22 Wenchang West Road, Wuhu, Anhui 241002, P.R. China

E-mail: 422627721@qq.com

E-mail: zhangyao@ahedu.gov.cn

Abbreviations: DMEM, Dulbecco's modified Eagle's medium; HSP70, heat shock protein 70; GFP-HSP70, green fluorescent protein-tagged HSP70; EMT, epithelial-mesenchymal transition; ROS, reactive oxygen species

Key words: salidroside, cell migration, reactive oxygen species, heat shock protein 70 , epithelial-mesenchymal transition dichloro-dihydro-fluorescein diacetate. The results indicated that salidroside significantly suppressed cell proliferation and colony formation, inhibited cell migration and invasion, increased E-cadherin expression and decreased $\mathrm{N}$-cadherin, MMP-2 and MMP-9 expression. Furthermore, salidroside suppressed ROS production and subsequently reduced the phosphorylation of Src, Akt, ERK and FAK. Salidroside also inhibited HSP70 expression, and HSP70 overexpression reversed the inhibitory effects of salidroside on BGC-823 cell proliferation, migration and invasion. In conclusion, the present study revealed that salidroside inhibited the proliferation, migration and invasion of BGC-823 cells by downregulating ROS-mediated Src-associated signaling pathway activation and HSP70 expression.

\section{Introduction}

Gastric cancer is the fourth most common cancer and the second most frequent cause of cancer-associated mortality worldwide (1-3). Although several aggressive treatment strategies, including surgery, radiotherapy and chemotherapy, are used to treat gastric cancer, the treatment outcomes for advanced gastric cancer remain unsatisfactory (4). The majority of patient mortalities are due to tumor recurrence and metastasis. At present, there are no effective therapeutic methods to prevent tumor recurrence and metastasis (5). Therefore, the development of effective and low toxicity drugs for the inhibition of tumor recurrence and metastasis is required.

Natural, biologically active products are widely used in clinical and basic research due to their low toxicity and often potent effects (6). At present, plant-derived anticancer drugs used clinically include vinblastine, vincristine, paclitaxel, and camptothecin $(7,8)$. Salidroside is a phenyl propanoid glycoside extracted from the flowering plant Rhodiola rosea. Salidroside has been reported to have various pharmacological actions, including anti-inflammatory $(9,10)$, anti-tumor (11), and neuroprotective effects (12). Growing evidence has demonstrated that salidroside significantly affects the proliferation, migration and apoptosis of various tumor types, including fibrosarcoma, breast, colon and bladder cancer (6,7,11-13). 
However, the effects of salidroside on gastric cancer cell proliferation, migration and invasion remain to be elucidated.

Reactive oxygen species (ROS) within cells have been identified as important second messengers in intracellular signaling cascades, which induce and maintain the oncogenic phenotype of cancer cells $(14,15)$. ROS are tumorigenic by virtue of their ability to increase cell proliferation, survival and cellular migration (15). Therefore, inhibiting the production of ROS may be exhibits anticancer effects in the treatment of tumour $(7,16,17)$.

Stress-inducible heat shock protein 70 (HSP70) is expressed at extremely low levels in cells under normal conditions and is not essential for life $(18,19)$. However, HSP70 is highly expressed in various tumor tissues, including breast cancer and melanoma. In addition, HSP70 expression is associated with cancer cell proliferation, apoptosis, metastasis, prognosis and differentiation (19). Therefore, inhibition of HSP70 expression may serve as an effective strategy in cancer therapy.

In the present study, the effects of salidroside on the proliferation, migration and invasion of BGC-823 cells and the potential molecular mechanisms were investigated. The results revealed that salidroside inhibited BGC-823 cell proliferation, migration and invasion in a dose-dependent manner, through the suppression of HSP70 expression, as well as ROS-mediated and proto-oncogene tyrosine-protein kinase Src (Src)-associated signaling pathways. The present study may provide a novel perspective for the application of salidroside in tumor therapy.

\section{Materials and methods}

Antibodies and reagents. Salidroside (purity, >98\%) was purchased from Sigma-Aldrich (Merck KGaA, Darmstadt, Germany). The antibodies against phosphorylated (p)-Src (Tyr416; cat. no. 6943s; 1:1,000), Src (cat. no. 2109s; 1:1,000), p-protein kinase B (Akt; Ser473; cat. no. 4060s; 1:500), Akt (cat. no. 9272s; 1:1,000), p-p44/42 mitogen-activated protein kinase 1 (p-ERK; Thr202/Tyr204; cat. no. 4376s; 1:1,000), ERK (cat. no. 9102s; 1:1,000), p-focal adhesion kinase 1 (FAK; Tyr576/577; cat. no. 3281s; 1:1,000), FAK (cat. no. 3285s; 1:1,000), HSP70 (cat. no. 4873s; 1:1,000), E-cadherin (cat. no. $3195 \mathrm{~s} ; 1: 1,000$ ), N-cadherin (cat. no. 4061s; $1: 1,000), \beta$-actin (cat. no. 4970s; 1:1,000) and GAPDH (cat. no. 5174s; 1:1,000) were obtained from Cell Signaling Technology, Inc., (Danvers, MA, USA). A Cell Counting Kit (CCK)-8 assay was purchased from Nanjing KeyGen Biotech Co., Ltd. (Nanjing, China). Matrix metalloproteinase (MMP)-2 and -9 enzyme-linked immunosorbent assay (ELISA) kits were obtained from ABclonal Biotech Co., Ltd., (Woburn, MA, USA).

Cell culture. The human gastric cancer cell line, BGC-823, was purchased from Guangzhou Jennio Biotech Co., Ltd., (Guangzhou, China). Cells were cultured in Dulbecco's modified Eagle's medium (DMEM; HyClone; GE Healthcare Life Sciences, Logan, UT, USA) supplemented with $10 \%$ fetal bovine serum (FBS; Gibco; Thermo Fisher Scientific, Inc. Waltham, MA, USA), $100 \mu \mathrm{g} / \mathrm{ml}$ streptomycin and $100 \mathrm{U} / \mathrm{ml}$ penicillin at $37^{\circ} \mathrm{C}$ in an environment containing $5 \% \mathrm{CO}_{2}$.

Cell viability assay. Cell viability was detected using a CCK-8 kit. In brief, BGC-823 cells were seeded onto a 96-well plate at a density of $1 \times 10^{4}$ cells/well. Following $12 \mathrm{~h}$ of culture at $37^{\circ} \mathrm{C}$, the cells were treated with different doses of salidroside $(25,50,100,200,400$ and $600 \mu \mathrm{g} / \mathrm{ml})$ for $24 \mathrm{~h}$ at $37^{\circ} \mathrm{C}$. CCK-8 reagent $(10 \mu \mathrm{l})$ was subsequently added to each well and the cells were incubated for a further $2 \mathrm{~h}$ at $37^{\circ} \mathrm{C}$ with $5 \% \mathrm{CO}_{2}$. The absorbance was detected using a Multiskan GO microplate spectrophotometer (Thermo Fisher Scientific, Inc.) at a wavelength of $450 \mathrm{~nm}$. Each treatment was repeated three times.

Colony formation assay. BGC-823 cells were incubated in a 6 -well culture plate at 2,000 cells/well. After treatment with $(200,400$ and $600 \mu \mathrm{g} / \mathrm{ml})$ or without salidroside for 6 days at $37^{\circ} \mathrm{C}$, the cell culture medium was discarded. Cells were subsequently fixed with $4 \%$ paraformaldehyde for $20 \mathrm{~min}$ at room temperature and stained with $0.1 \%$ crystal violet for $30 \mathrm{~min}$ at room temperature. Following this, cells were washed 3 times in PBS and colonies containing $>15$ cells were counted under a fluorescence inverted microscope (magnification, x100), 5 fields of view were assessed, (Olympus Corporation, Tokyo, Japan) and images were captured.

Scratch wound-healing assay. BGC-823 cells were seeded in a 12 -well cell culture plate and cultured at $37^{\circ} \mathrm{C}$ to full confluence. The confluent cell monolayers were wounded by scratching with a pipette tip. Damaged cells were removed by washing with PBS and remaining cells in the plate were treated with salidroside $(600 \mu \mathrm{g} / \mathrm{ml})$ and incubated with DMEM with $10 \% \mathrm{FBS}$ for $24 \mathrm{~h}$ at $37^{\circ} \mathrm{C}$. Images were captured of cell migration over the injured area at 0 and $24 \mathrm{~h}$ using fluorescence inverted microscope (magnification, x40; Olympus Corporation).

Cell migration and invasion assay. Cell migration was analyzed in 24-well cell culture plates with a Transwell membrane and $8-\mu \mathrm{m}$ pore filter inserts (EMD Millipore, Billerica, MA, USA). To examine invasion, wells were coated with Matrigel (BD Biosciences, Franklin Lakes, NJ, USA). Briefly, BGC-823 cells were harvested following treatment with 200,400 and $600 \mu \mathrm{g} / \mathrm{ml}$ salidroside for $24 \mathrm{~h}$ at $37^{\circ} \mathrm{C}$ and suspended in serum-free DMEM. Cells $\left(2 \times 10^{4}\right.$ cells/well) were added to the upper chamber and $0.6 \mathrm{ml}$ DMEM containing $20 \%$ FBS was added to the lower chamber. After incubation for $24 \mathrm{~h}$ in the $5 \% \mathrm{CO}_{2}$ incubator at $37^{\circ} \mathrm{C}$, cells that had migrated to the lower surface of the membrane were fixed with $4 \%$ paraformaldehyde for $20 \mathrm{~min}$ at room temperature. The membrane was washed with PBS three times and cells were subsequently stained with $0.1 \%$ crystal violet for $30 \mathrm{~min}$ at room temperature. The cells remaining on the upper surface were wiped away gently with a cotton swab. Images of the migrated cells were captured by fluorescence inverted microscope (magnification, x100; Olympus Corporation).

Measurement of intracellular ROS. Intracellular ROS levels were determined with a ROS assay kit (Beyotime Institute of Biotechnology, Haimen, China). Briefly, BGC-823 cells were treated with $600 \mu \mathrm{g} / \mathrm{ml}$ salidroside for $24 \mathrm{~h}$ at $37^{\circ} \mathrm{C}$ and subsequently incubated with $10 \mu \mathrm{mol} / 1$ dichloro-dihydro-fluorescein diacetate (DCFH-DA) for $30 \mathrm{~min}$ at $37^{\circ} \mathrm{C}$ in the dark. Cells 
A

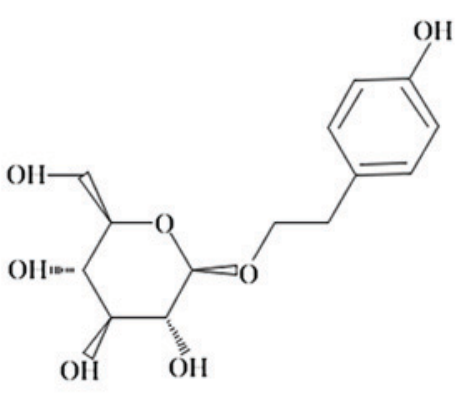

B

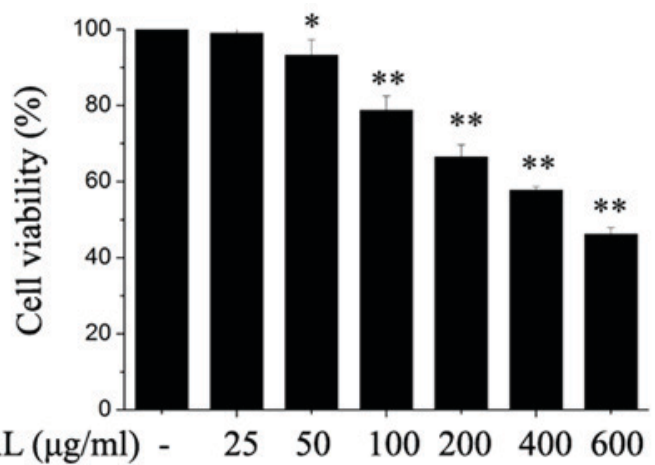

C

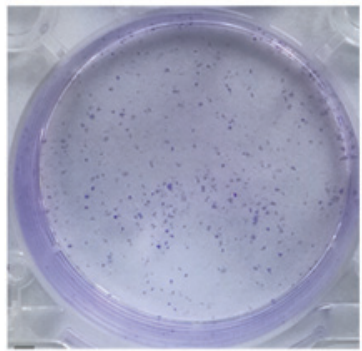

SAL $(200 \mu \mathrm{g} / \mathrm{ml})$

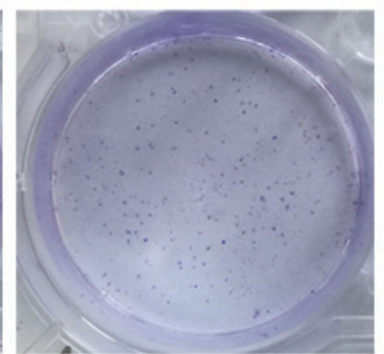

SAL $(400 \mu \mathrm{g} / \mathrm{ml})$

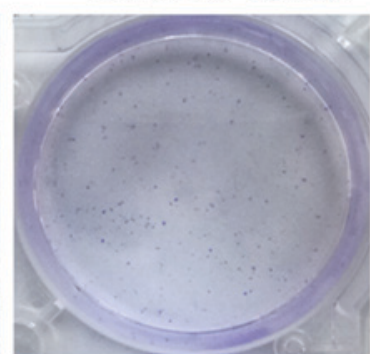

SAL $(600 \mu \mathrm{g} / \mathrm{ml})$

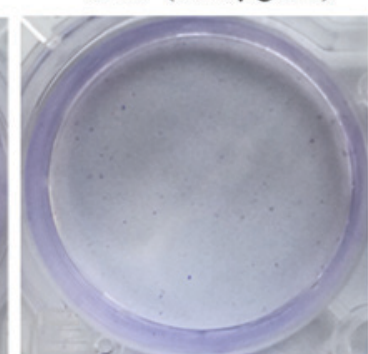

Figure 1. Salidroside inhibits the viability and colony formation of BGC-823 cells. (A) The chemical structure of salidroside. Cells were treated with different doses of salidroside for $24 \mathrm{~h}$. (B) Cell viability was detected with a Cell Counting Kit-8 and (C) the colony forming ability of BGC-823 cells was determined. Each treatment was repeated three times. ${ }^{*} \mathrm{P}<0.05,{ }^{* *} \mathrm{P}<0.01$ vs. control. SAL, salidroside.

were washed with PBS prior to examination by fluorescence microscopy (magnification, x100; Olympus Corporation).

Western blotting. Cells were lysed in radioimmunoprecipitation assay buffer containing proteinase inhibitors (Beyotime Institute of Biotechnology) for $30 \mathrm{~min}$ on ice. The protein was quantified by BCA/Bradford assay. An equal amount of protein $(50 \mu \mathrm{g})$ was loaded and separated with $12 \%$ SDS-PAGE, and then the proteins were transferred onto a nitrocellulose membrane, which was blocked with $5 \%$ skim milk powder for $1 \mathrm{~h}$ at room temperature. The membrane was washed with PBS three times and incubated with the indicated primary antibodies at $4^{\circ} \mathrm{C}$ overnight, followed by washing the membrane again with PBS three times. The membrane was subsequently incubated with IRdye $800 \mathrm{CW}$-conjugated IgG secondary antibody (cat. no. 926-32210; 1:5,000; LI-COR Biosciences, Lincoln, NE, USA) for $1 \mathrm{~h}$ at room temperature in the dark. The proteins were visualized using an Odyssey infrared imaging system (LI-COR Biosciences).

Cell transfection. Green fluorescent protein (GFP)-labeled HSP70 overexpression plasmids and negative plasmids (supplier: CMV-MCS-EGFP-SV40-Neomycin) were purchased from Shanghai GeneChem Co., Ltd. (Shanghai, China). BGC-823 cells $\left(2 \times 10^{5}\right)$ were transfected with $2 \mu \mathrm{g}$ HSP70 overexpression and negative plasmids for $24 \mathrm{~h}$ with Lipofectamine $^{\circledR} 3000$ reagent (Invitrogen; Thermo Fisher Scientific, Inc.) according to the manufacturer's protocol.

ELISA assay. MMP-2 (cat. no. RK00309) and MMP-9 (cat. no. RK00217) protein levels were detected in BGC-823 cells $\left(1 \times 10^{6}\right)$ with ELISA kits (ABclonal Biotech Co., Ltd.) according to the manufacturer's protocol. Analysis of each group was repeated three times.

Statistical analysis. Each experiment was repeated three times. Data were presented as the mean \pm standard deviation. SPSS software, version 17.0 (SPSS, Inc., Chicago, IL, USA) was used to perform statistical analysis. The results were compared using one-way analysis of variance followed by a post hoc Tukey test for multiple comparisons. $\mathrm{P}<0.05$ was considered to indicate a statistically significant difference.

\section{Results}

Salidroside inhibits the proliferation and colony formation of BGC-823 cells. BGC-823 cells were treated with different doses of salidroside (Fig. 1A) for $24 \mathrm{~h}$, followed by the addition of CCK-8 at $10 \mu \mathrm{l} /$ well. Following incubation for another $2 \mathrm{~h}$, cell viability was detected. Salidroside significantly suppressed BGC-823 cell viability and the inhibitory effect was dose-dependent (Fig. 1B). According to these results, salidroside concentrations of 200, 400 and $600 \mu \mathrm{g} / \mathrm{ml}$ were selected for the subsequent experiments. The effects of salidroside on the colony formation of BGC-823 cells were also determined. It was demonstrated that salidroside clearly inhibited BGC-823 cell colony formation in a dose-dependent manner (Fig. 1C).

Salidroside suppresses the migration and invasion of BGC-823 cells. A scratch wound healing assay was used to detect the migration of BGC-823 cells (Fig. 2A). It was demonstrated that wound closure in cells treated with salidroside $(600 \mu \mathrm{g} / \mathrm{ml})$ was significantly reduced compared with the control cells. A 
A

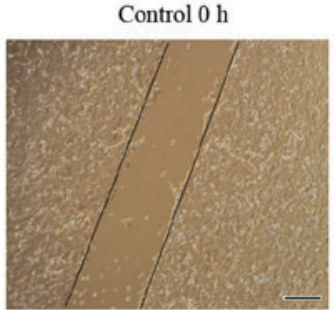

SAL $(600 \mu \mathrm{g} / \mathrm{ml}) 0 \mathrm{~h}$

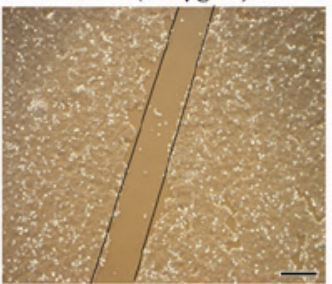

B

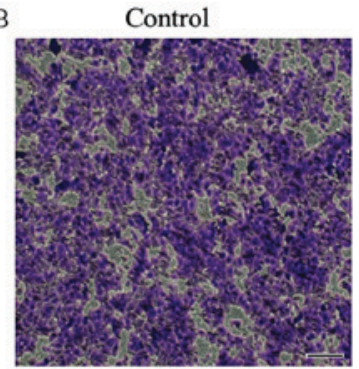

$\mathrm{C}$
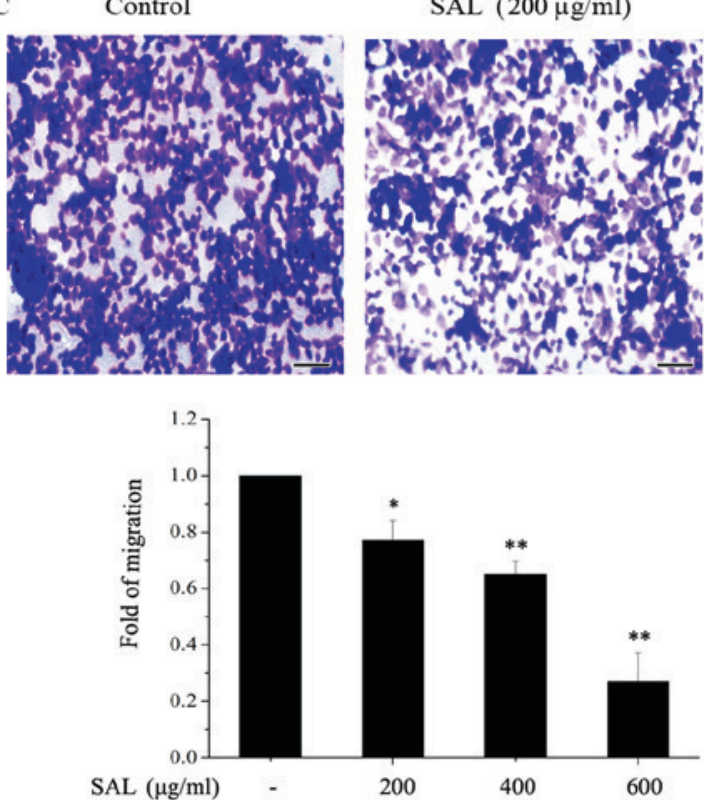

Control $24 \mathrm{~h}$

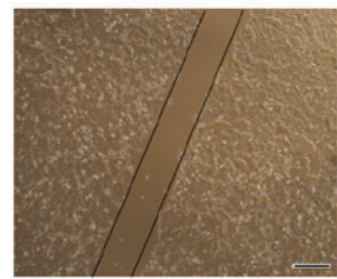

SAL $(600 \mu \mathrm{g} / \mathrm{ml}) 24 \mathrm{~h}$

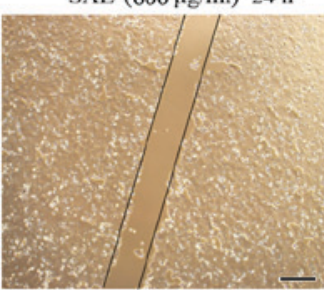

$\operatorname{SAL}(200 \mu \mathrm{g} / \mathrm{ml})$

SAL $(200 \mu \mathrm{g} / \mathrm{ml})$

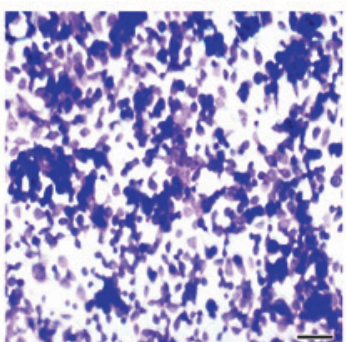

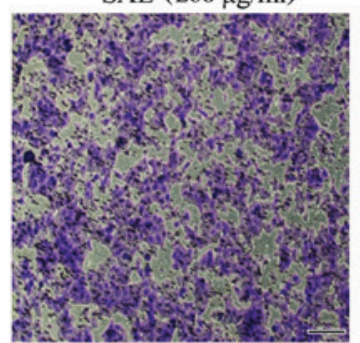

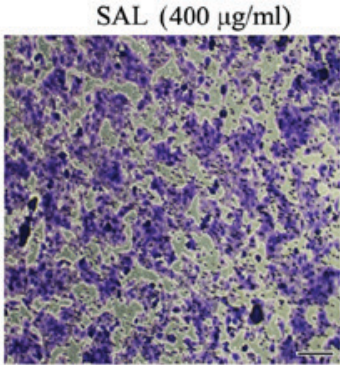

SAL $(400 \mu \mathrm{g} / \mathrm{ml})$

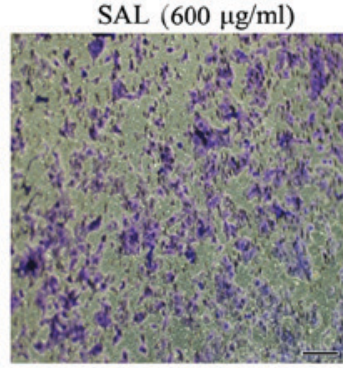

SAL $(600 \mu \mathrm{g} / \mathrm{ml})$
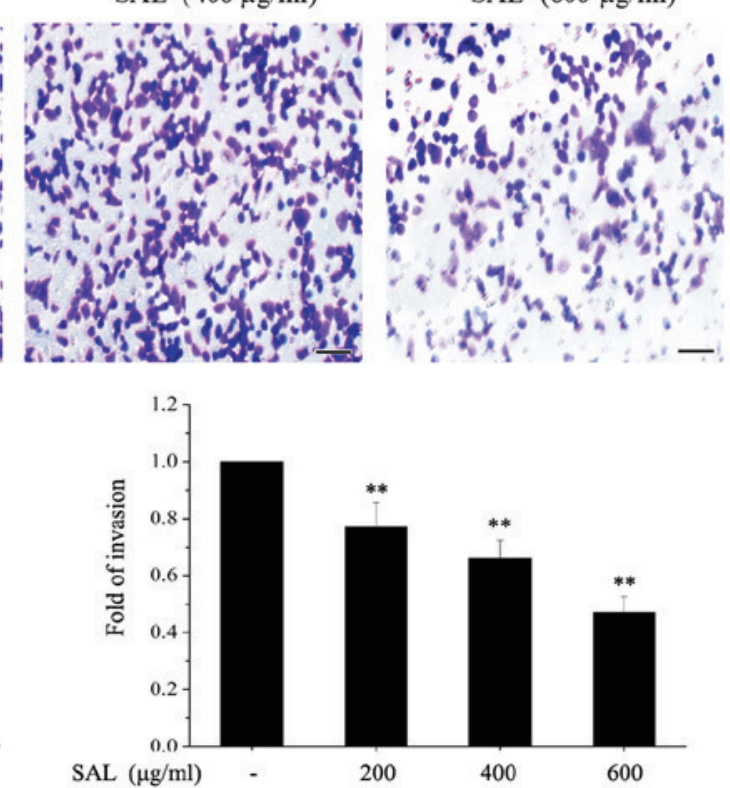

Figure 2. Salidroside reduces the migration and invasion of BGC-823 cells. (A) Confluent BGC-823 cells were wounded with a pipette tip and cell migration over the scraped area was analyzed. Scale bar, $250 \mu \mathrm{m}$. (B) Migration and (C) invasion of BGC-823 cells was detected by a Transwell assay. Scale bar, $50 \mu \mathrm{m}$. Each experiment was repeated three times. ${ }^{*} \mathrm{P}<0.05,{ }^{* *} \mathrm{P}<0.01$ vs. control. $\mathrm{SAL}$, salidroside.

Transwell assay was used to determine the migration and invasion of BGC-823 cells. The migration (Fig. 2B) and invasion (Fig. 2C) of BGC-823 cells treated with salidroside (200, 400 and $600 \mu \mathrm{g} / \mathrm{ml}$ ) for $24 \mathrm{~h}$ was markedly inhibited compared with the control cells, in a dose-dependent manner.

Salidroside reduces the levels of ROS, MMP-2 and MMP-9 in BGC-823 cells conditioned medium. As MMPs have an important role in tumor cell invasion (20), the effects of salidroside on the levels of MMP-2 and MMP-9 levels were detected by ELISA. Salidroside significantly reduced the levels of MMP-2 (Fig. 3A) and MMP-9 (Fig. 3B) in the treated medium of BGC-823 cells.

As oxidative stress may induce tumor cell migration and invasion through MMP upregulation (20), the effects of salidroside on ROS levels in BGC-823 cells were investigated. 
A

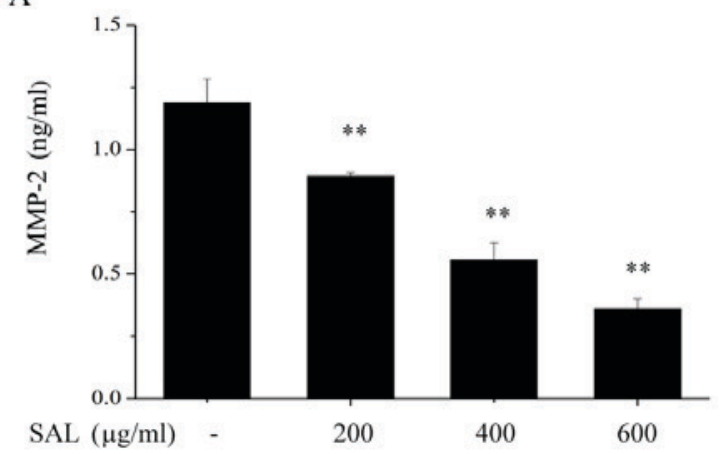

$\mathrm{C}$

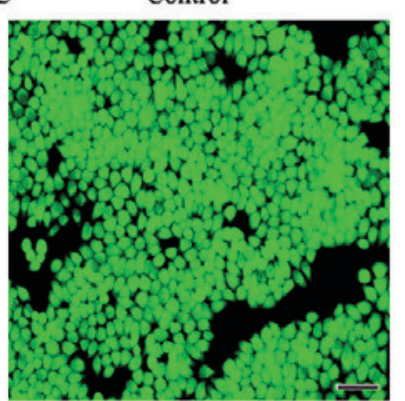

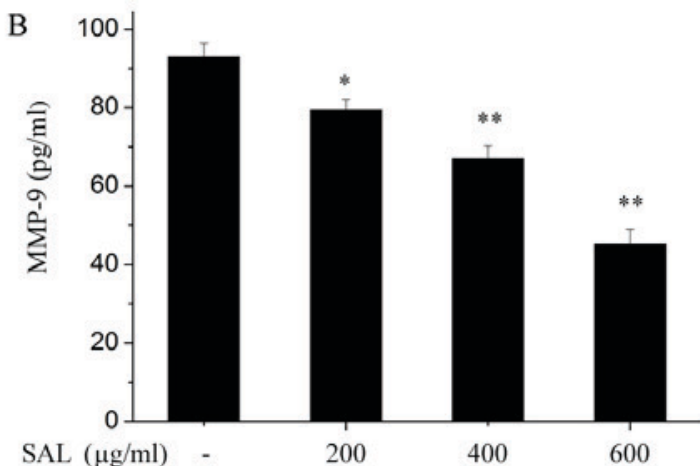

SAL $(\mu \mathrm{g} / \mathrm{ml})$
SAL $400 \mu \mathrm{g} / \mathrm{ml}$

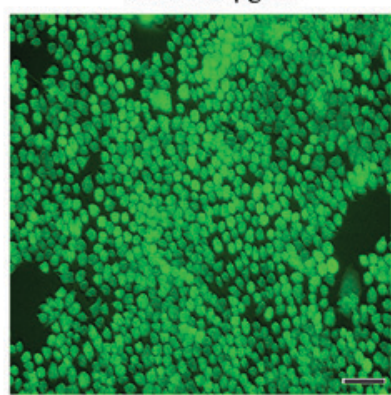

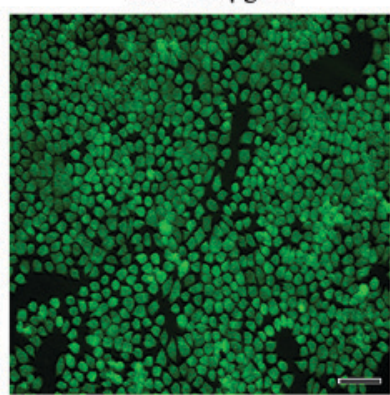

SAL $600 \mu \mathrm{g} / \mathrm{ml}$

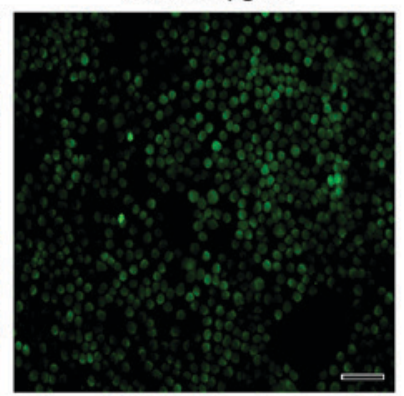

Figure 3. Salidroside decreases the levels of MMP-2, MMP-9 and ROS generation in BGC-823 cells. BGC-823 cells were treated with salidroside for $24 \mathrm{~h}$. (A) MMP-2 and (B) MMP-9 levels were detected by enzyme-linked immunosorbent assay. (C) Intracellular ROS generation was detected by a ROS kit. Scale bar, $50 \mu \mathrm{m} .{ }^{*} \mathrm{P}<0.05,{ }^{* *} \mathrm{P}<0.01$ vs. control. MMP, matrix metalloproteinase; ROS, reactive oxygen species; SAL, salidroside.

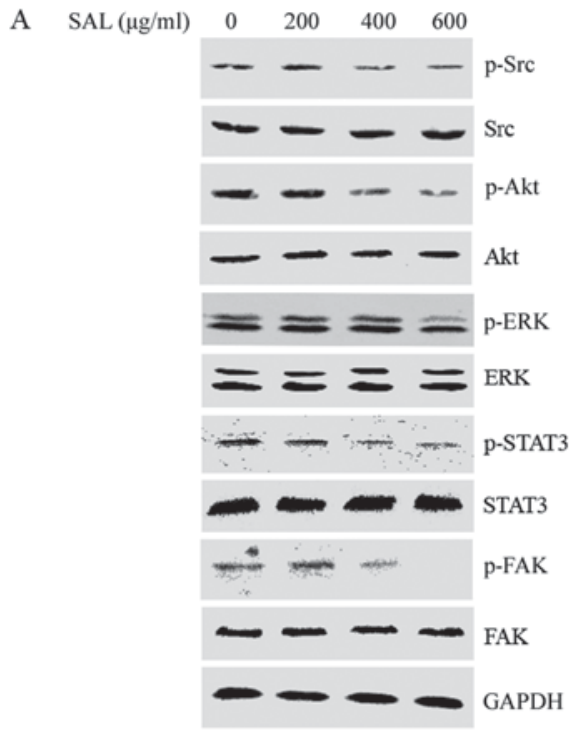

B $\operatorname{SAL}(\mu \mathrm{g} / \mathrm{ml}) \quad 0 \quad 200 \quad 400 \quad 600$

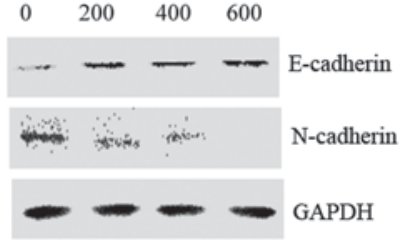

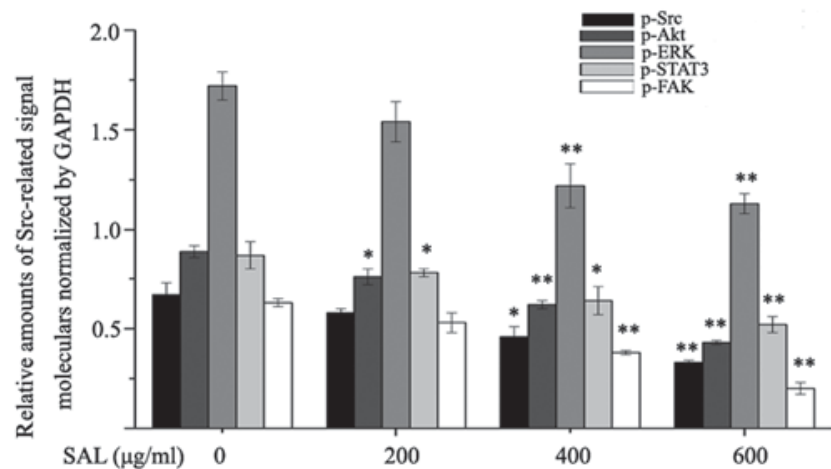

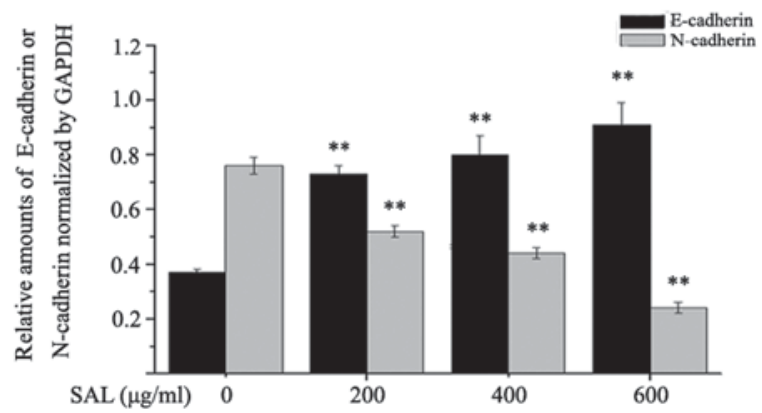

Figure 4. Salidroside affects the phosphorylation of Src-associated signaling pathway proteins and epithelial-mesenchymal transition marker expression. BGC-823 cells were treated with different concentrations of salidroside for $24 \mathrm{~h}$ and (A) the phosphorylation of Src, Akt, STAT3, ERK and FAK, in addition to (B) the levels of E-cadherin and $\mathrm{N}$-cadherin were detected by western blot analysis. ${ }^{*} \mathrm{P}<0.05,{ }^{* *} \mathrm{P}<0.01$ vs. control. p, phosphorylated; Akt, protein kinase B; Src, proto-oncogene tyrosine-protein kinase Src; STAT3, signal transducer and activator of transcription 3; ERK, mitogen-activated protein kinase 1; FAK, focal adhesion kinase 1; SAL, salidroside. 
A
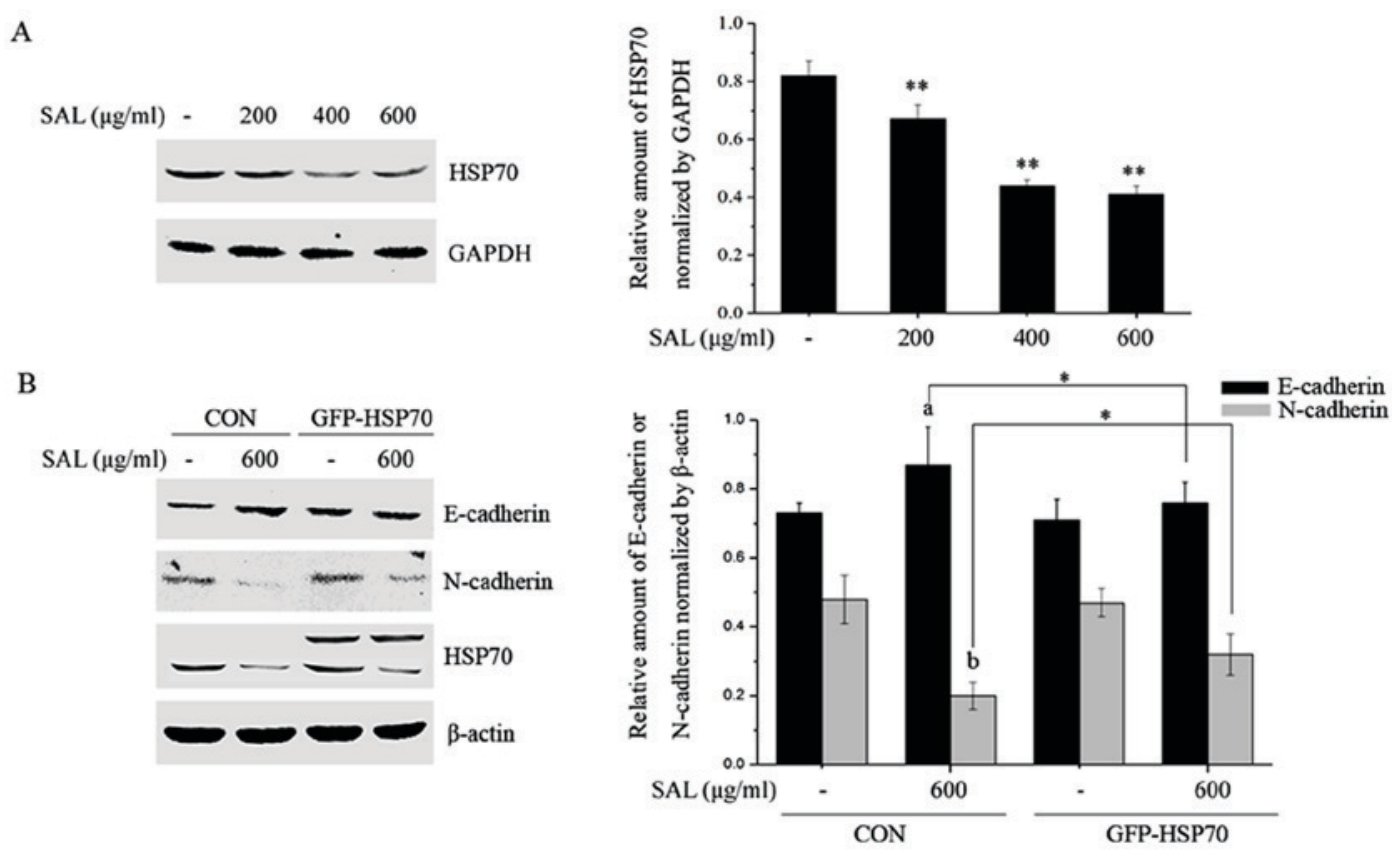

Figure 5. Salidroside attenuates HSP70 expression and HSP70 overexpression rescues cells from the effects of salidroside on epithelial-mesenchymal transition marker expression. (A) BGC-823 cells were treated with salidroside for $24 \mathrm{~h}$. The total proteins were extracted and HSP70 expression was detected by western blotting. ${ }^{* *} \mathrm{P}<0.01$ vs. control. (B) BGC-823 cells were transfected with control and HSP70 overexpression plasmids for $24 \mathrm{~h}$ and subsequently treated with or without salidroside for a further $24 \mathrm{~h}$. Total proteins were collected and the expression of E-cadherin, N-cadherin and HSP70 proteins was detected by western blotting. In the blot probed with anti-HSP70, the upper band was the overexpressed HSP70 with a GFP tag and the lower band was endogenous HSP70. The experiments were done in triplicate and data are presented as the mean \pm standard deviation. ${ }^{\mathrm{a}} \mathrm{P}<0.05$ vs. control plasmid transfected cells, ${ }^{\mathrm{b}} \mathrm{P}<0.01$ compared with the control plasmids transfected cells, ${ }^{*} \mathrm{P}<0.05$ (as indicated). HSP70, heat shock protein 70; GFP, green fluorescent protein; SAL, salidroside.

Intracellular ROS levels were detected with DCFH-DA. As presented in Fig. 3C, salidroside treatment clearly reduced the intracellular ROS levels in a dose-dependent manner.

Salidroside inhibits the expression of epithelial-mesenchymal transition (EMT) markers and the phosphorylation of Src-associated signaling pathway proteins in BGC-823 cells. A previous study reported that Src activation governs a variety of signaling pathways, including proliferation, migration and invasion, through various proteins, including Akt, signal transducer and activator of transcription (STAT)3, ERK and FAK $(21,22)$. Therefore, the effects of salidroside on Src-associated signaling pathways were investigated. As presented in Fig. 4A, salidroside treatment inhibited the phosphorylation of Src, Akt, STAT3, ERK and FAK in a dose-dependent manner. EMT is thought to be involved in the migration and invasion of tumor cells (23). Therefore, the effects of salidroside on the expression of EMT markers were examined. The protein expression levels of the epithelial marker E-cadherin and the mesenchymal marker $\mathrm{N}$-cadherin were detected in BGC-823 cells treated with different concentrations of salidroside for $24 \mathrm{~h}$. Salidroside treatment significantly reduced the level of $\mathrm{N}$-cadherin and enhanced the expression of E-cadherin (Fig. 4B). The aforementioned results suggested that salidroside may have inhibited the proliferation, migration and invasion of BGC-823 cells through its effects on EMT via Src-associated signaling pathways.

Salidroside attenuates HSP70 expression and HSP70 overexpression rescues cells from the effects of salidroside on EMT markers. HSP70 is highly expressed in various tumor tissues and its expression is positively correlated with cell proliferation and metastasis (19); therefore, the effects of salidroside on HSP70 expression were investigated. BGC-823 cells were treated with different doses of salidroside for $24 \mathrm{~h}$, total proteins were collected and the HSP70 levels were determined by western blotting. Salidroside significantly attenuated the expression of HSP70 (Fig. 5A). Subsequently, BGC-823 cells were transfected with negative and HSP70 overexpression plasmids and the role of HSP70 in salidroside-induced EMT marker expression was investigated. As presented in Fig. 5B, salidroside treatment significantly enhanced levels of E-cadherin and reduced the $\mathrm{N}$-cadherin levels in negative plasmid transfected BGC-823 cells. The alterations in E-cadherin and $\mathrm{N}$-cadherin expression induced by salidroside were partially prevented by HSP70 overexpression.

HSP70 suppresses the effects of salidroside on colony formation, migration and invasion in BGC-823 cells. The effect of HSP70 on colony formation, migration and invasion was investigated. BGC-823 cells were transfected with control or HSP70 overexpression plasmids for $24 \mathrm{~h}$. Following transfection, cells were treated with salidroside for a further $24 \mathrm{~h}$ and colony formation, migration and invasion were subsequently examined. As presented in Fig. 6A and B, it was demonstrated that salidroside significantly suppressed the migration and invasion of BGC-823 cells in control plasmid transfected BGC-823 cells. However, the inhibitory effects induced by salidroside were prevented in BGC-823 cells transfected with HSP70 overexpression plasmid. Salidroside significantly attenuated colony formation in control plasmid transfected BGC-823 cells (Fig. 6C). This inhibitory effect 

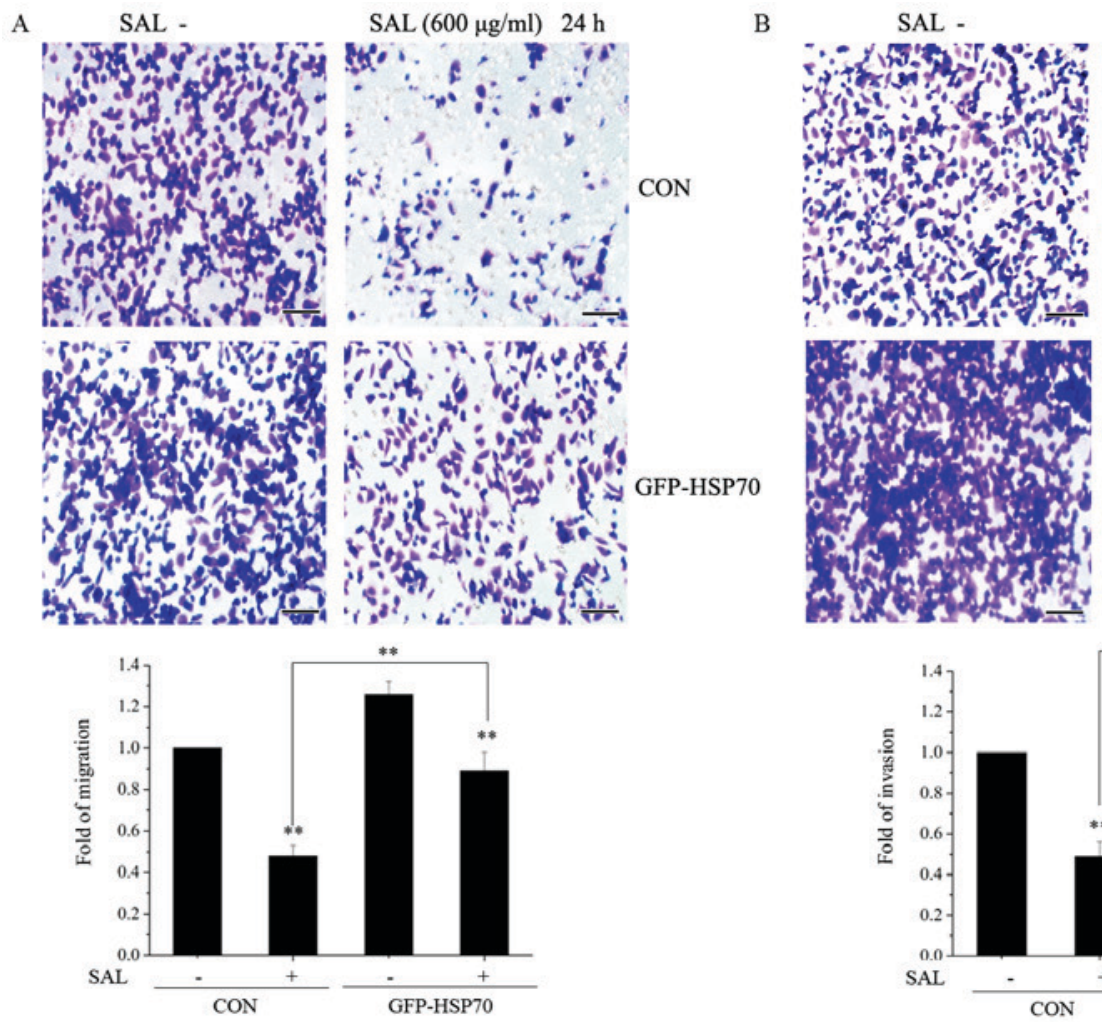

$\operatorname{SAL}(600 \mu \mathrm{g} / \mathrm{ml}) 24 \mathrm{~h}$

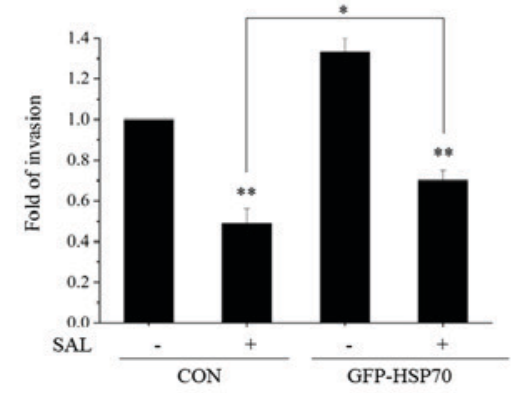

C
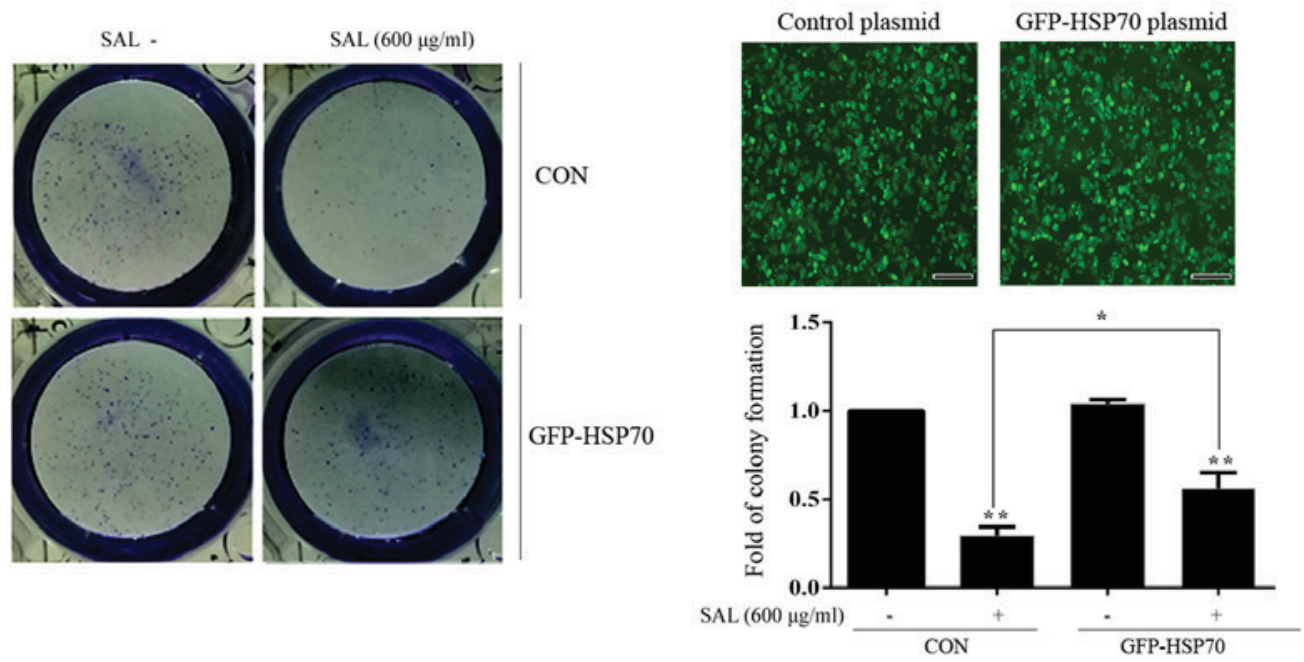

Figure 6. HSP70 suppresses the effects of salidroside on colony formation, migration and invasion in BGC-823 cells. BGC-823 cells were transfected with negative control and HSP70 overexpression plasmids for $24 \mathrm{~h}$. Following transfection, cells were treated with salidroside for a further $24 \mathrm{~h}$ and the (A) migration and (B) invasion of cells was investigated by a Transwell assay. Scale bar, $50 \mu \mathrm{m}$. (C) The colony forming abilityof cells was detected. Experiments were performed three times. " $\mathrm{P}<0.05$ (as indicated); ${ }^{* *} \mathrm{P}<0.01$ vs. control plasmid transfected cells. HSP70, heat shock protein 70; GFP, green fluorescent protein; SAL, salidroside; $\mathrm{CON}$, control.

was reversed by HSP70 overexpression. Taken together, these results suggested that $\mathrm{HSP} 70$ may have antagonized the effects of salidroside on BGC-823 cell proliferation and migration. Taken together, the results demonstrated that the inhibition of HSP70 expression may be a molecular mechanism by which salidroside suppresses BGC-823 cell proliferation, migration and invasion.

\section{Discussion}

Salidroside has been reported to have anti-tumor effects in vitro and in vivo $(11,20)$. In the present study, it was demonstrated that salidroside inhibited the proliferation, colony formation, migration and invasion of BGC-823 cells. The potential mechanisms may be associated with the inhibitory effects of salidroside on ROS-mediated and Src-associated signaling pathways, as well as HSP70 expression.

Inhibition of tumor growth is an important aim in all strategies used to prevent tumor progression. Dysregulated cell proliferation is a hallmark of cancer development (24). In the present study, it was confirmed that salidroside, a bioactive component extracted from Rhodiola rosea, had an inhibitory effect on the cell viability of BGC-823 cells through a CCK-8 assay, and this inhibitory effect was concentration-dependent. 
In addition, salidroside was demonstrated to inhibit BGC-823 cell colony formation, particularly at a dose of $600 \mu \mathrm{g} / \mathrm{ml}$.

Increasing evidence has revealed that salidroside inhibits the migration and invasion of various tumors $(7,20)$. Consistent with these findings, salidroside treatment significantly suppressed BGC-823 cell migration and invasion in the present study, in a dose-dependent manner. MMPs have a key role in tumor cell invasion, migration and tumor angiogenesis (25). MMPs are a family of proteolytic enzymes that facilitate tumor cell migration by degrading the basement membrane and other components of the extracellular matrix (26). MMP-2 and MMP-9 are important members of the MMP family. Downregulation of MMP-2 and MMP-9 expression inhibits cancer cell invasion and metastasis $(27,28)$. Previous reports have demonstrated that salidroside markedly suppresses MMP-2 and MMP-9 expression $(7,20)$. Consistent with these results, the present study demonstrated that salidroside decreased MMP-2 and MMP-9 expression in BGC-823 cells, suggesting that salidroside may have reduced the metastatic capabilities of BGC-823 cells via suppression of MMP-2 and MMP-9 expression. As oxidative stress may induce tumorcell migration and invasion through the upregulation of MMP expression (20), the effects of salidroside on ROS levels were investigated. It was revealed that salidroside treatment significantly inhibited intracellular ROS generation in a dose-dependent manner.

EMT is a fundamental biological process in which epithelial cells undergo a dramatic remodeling of the cytoskeleton, lose basal-apical polarity and acquire an increased capacity to metastasize to distant organs (29-31). Alterations in the expression of EMT-associated markers, including a decrease in E-cadherin and an increase in $\mathrm{N}$-cadherin, are closely associated with the invasive and metastatic capacity of cancer cells (29). The results of the present study confirmed that salidroside treatment enhanced the expression of E-cadherin and reduced the expression of $\mathrm{N}$-cadherin.

A previous study indicated that Src activation governs several pathways, including those involved in survival, angiogenesis, proliferation, migration and invasion, through a variety of proteins, including phosphatidiylinositol-4,5-biphosphate 3-kinase (PI3K)/Akt, STAT3, ERK and FAK (32). In addition, Src activation is associated with an epidermal to mesenchymal-like transition in models of epithelial cancer (22). Therefore, the effects of salidroside on the Src-associated signaling pathway proteins were further investigated in the current study. Salidroside treatment significantly inhibited the phosphorylation of Src, Akt, ERK, STAT3 and FAK, suggesting that salidroside may have inhibited the migration and invasion of BGC-823 cells via suppression of ROS-mediated Src-associated signaling pathway activation.

One of the most effective markers for the detection of tumor cells is HSP70. HSP70 expression is positively correlated with cell proliferation and metastasis. Therefore, inhibition of HSP70 expression may serve as a target for cancer therapy (19). In the present study, whether the inhibitory effects of salidroside on migration, invasion and proliferation were partly associated with HSP70 expression was investigated. The results revealed that salidroside treatment significantly attenuated HSP70 expression. Overexpression of HSP70 reversed the inhibitory

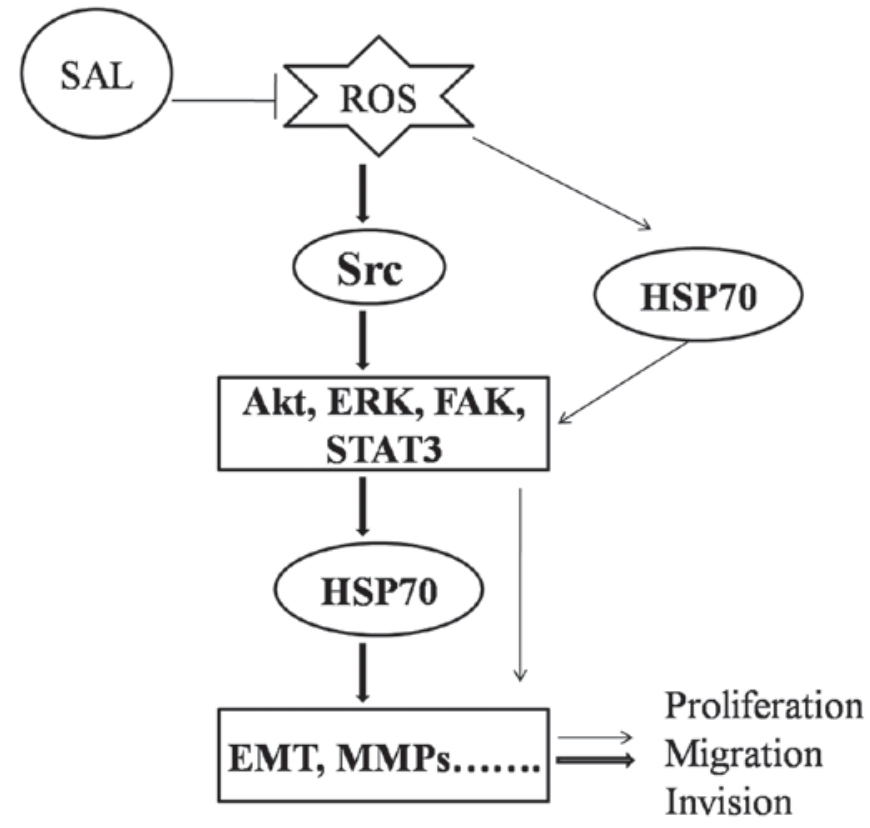

Figure 7. Schematic diagram illustrating the signaling pathways involved in the inhibitory effect of salidroside on biological function, via Src-associated signaling pathways and HSP70 expression. SAL, salidroside; ROS, reactive oxygen species; Src, proto-oncogene tyrosine-protein kinase Src; HSP70, heat shock protein 70; Akt, protein kinase B; STAT3, signal transducer and activator of transcription 3; ERK, mitogen-activated protein kinase 1; FAK, focal adhesion kinase 1; EMT, epithelial-mesenchymal transition; MMP, matrix metalloproteinase.

effects of salidroside on EMT marker expression, as well as the proliferation, migration and invasion of BGC-823 cells. Taken together, these results suggested that HSP70 expression downregulation may be another possible molecular mechanism by which salidroside suppressed the proliferation, migration and invasion of BGC-823 cells. Transforming growth factor (TGF)- $\beta /$ mothers against decapentaplegic homologs (Smads) signaling is thought to be important in the promotion of EMT in human breast cancer (33). Additionally, a previous report revealed that HSP70 inhibits high glucose-induced EMT by modulating Smad expression and activation in peritoneal mesothelial cells (34). Furthermore, HSP70 increases the cellular defense capacity through inhibition of TGF- $\beta /$ Smad and ERK signaling pathways, thereby protecting peritoneal mesothelial cells from advanced glycation end products-induced EMT (35). Collectively, these reports suggest that the potential molecular mechanism by which HSP70 regulates EMT may be through Smad signaling pathways. However, whether HSP70 regulated EMT in BGC-823 cells through Smad is still unclear. In the future, the authors of the present study will investigate the effect of HSP70 on Smad signaling pathway activation in gastric cancer.

The present study indicated that HSP70 and Src-associated signaling pathways may have contributed to the proliferation and metastasis of BGC- 823 cells. However, the association between HSP70 and these pathways requires further clarification. Resveratrol has been reported to downregulate HSP70 expression through modulation of Akt and ERK1/2 pathways in chronic myelogenous leukemia cells (36). In addition, it has been demonstrated that the PI3K/Akt signaling pathway is involved in the induction of HSP70 expression and that blockade 
of the PI3K/Akt pathway enhances the sensitivity of Raji cells to chemotherapy through downregulation of HSP70 (37). Banerjee Mustafi et al (38) reported that heat stress upregulates the expression of HSP70 through a ROS-mediated p38 mitogen activated protein kinase-Akt signaling pathway. Furthermore, Src activation governs a variety of pathways, including PI3K/Akt, STAT3, ERK and FAK (32). Therefore, based on the aforementioned results, it was hypothesized that a potential mechanism by which salidroside inhibited the proliferation and migration of BGC-823 cells in the present study may be through HSP70 downregulation via suppression of ROS-mediated Src-associated signaling pathway activation (Fig. 7).

Budina-Kolomets et al (18) revealed that p-FAK is a client protein of HSP70, and inhibition of HSP70 may suppress FAK-dependent invasion in human melanoma cells (18). In addition, Diao et al (39) reported that exosomal HSP70 expression triggers STAT3 phosphorylation in myeloid-derived suppressor cells. Based on these findings and the results of the present study, it was theorized that salidroside may have also inhibited the proliferation and migration of BGC-823 cells through the downregulation of HSP70 expression, followed by suppression of the Src-mediated phosphorylation of FAK and STAT3 (Fig. 7). However, the present study was unable to obtain clear evidence of the association between HSP70 and Src-associated signaling, which will be investigated in future research.

In conclusion, the results of the present study demonstrated that salidroside significantly inhibited BGC-823 cell proliferation, migration and invasion. Additionally, salidroside treatment inhibited ROS-mediated Src-associated signaling pathway protein phosphorylation and HSP70 expression. Taken together, these data suggested that salidroside suppressed the proliferation, migration and invasion of BGC-823 cells, at least partially through ROS-activated Src-associated signaling pathways and HSP70. The present study provides novel insights into the antitumor effects of salidroside in gastric cancer.

\section{Acknowledgements}

Not applicable.

\section{Funding}

This study was supported by the National Nature Science Foundation of China (grant no. 81601380), Natural Science Research Project of Anhui Colleges and Universities (grant no. KJ2016SD59), Outstanding Young Talent Support Program Key Projects in Anhui Colleges and Universities (grant no. gxyqZD2016173) and Anhui Province Key Laboratory of Active Biological Macromolecules (grant no. 1306C083008).

\section{Availability of data and materials}

The data and materials during the current study are available from the corresponding author on reasonable request.

\section{Author's contributions}

Conceived and designed the experiments: ZQ and YZ. Performed the experiments: ZQ, TT, LS and YM. Analyzed the data: SQ, LL, YL and LY. Wrote the paper: ZQ.

\section{Ethics approval and consent to participate}

Not applicable.

\section{Consent for publication}

Not applicable.

\section{Competing interests}

The authors declare that they have no competing interests.

\section{References}

1. Zhang L, Xu Z, Xu X, Zhang B, Wu H, Wang M, Zhang X, Yang T, Cai J, Yan Y, et al: SALL4, a novel marker for human gastric carcinogenesis and metastasis. Oncogene 33: 5491-5500, 2014.

2. Wang L, Chen X, Du Z, Li G, Chen M, Liang G and Chen T: Curcumin suppresses gastric tumor cell growth via ROS-mediated DNA polymerase gamma depletion disrupting cellular bioenergetics. J Exp Clin Cancer Res 36: 47, 2017.

3. Brenner H, Rothenbacher D and Arndt V: Epidemiology of stomach cancer. Methods Mol Biol 472: 467-477, 2009.

4. van Hagen P, Hulshof MC, van Lanschot JJ, Steyerberg EW, van Berge Henegouwen MI, Wijnhoven BP, Richel DJ, Nieuwenhuijzen GA, Hospers GA, Bonenkamp JJ, et al: Preoperative chemoradiotherapy for esophageal or junctional cancer. N Engl J Med 366: 2074-2084, 2012.

5. Karimi P, Islami F, Anandasabapathy S, Freedman ND and Kamangar F: Gastric cancer: Descriptive epidemiology, risk factors, screening, and prevention. Cancer Epidemiol Biomarkers Prev 23: 700-713, 2014.

6. Sun KX, Xia HW and Xia RL: Anticancer effect of salidroside on colon cancer through inhibiting JAK2_STAT3 signaling pathway. Int J Clin Exp Pathol 8: 615-621, 2015.

7. Zhao G, Shi A, Fan Z and Du Y: Salidroside inhibits the growth of human breast cancer in vitro and in vivo. Oncol Rep 33: 2553-2560, 2015.

8. Paterson I and Anderson EA: Chemistry. The renaissance of natural products as drug candidates. Science 310: 451-453, 2005.

9. Qi Z, Qi S, Ling L, Lv J and Feng Z: Salidroside attenuates inflammatory response via suppressing JAK2-STAT3 pathway activation and preventing STAT3 transfer into nucleus. Int Immunopharmacol 35: 265-271, 2016.

10. Guan S, Feng H, Song B, Guo W, Xiong Y, Huang G, Zhong W, Huo M, Chen N, Lu J and Deng X: Salidroside attenuates LPS-induced pro-inflammatory cytokine responses and improves survival in murine endotoxemia. Int Immunopharmacol 11: 2194-2199, 2011.

11. Fan XJ, Wang Y, Wang L and Zhu M: Salidroside induces apoptosis and autophagy in human colorectal cancer cells through inhibition of PI3K/Akt/mTOR pathway. Oncol Rep 36: 3559-3567, 2016.

12. Zhang L, Ding W, Sun H, Zhou Q, Huang J, Li X, Xie Y and Chen J: Salidroside protects PC12 cells from MPP(+)-induced apoptosis via activation of the PI3K/Akt pathway. Food Chem Toxicol 50: 2591-2597, 2012.

13. Liu Z, Li X, Simoneau AR, Jafari M and Zi X: Rhodiola rosea extracts and salidroside decrease the growth of bladder cancer cell lines via inhibition of the mTOR pathway and induction of autophagy. Mol Carcinog 51: 257-267, 2012.

14. Forman HJ, Torres M and Fukuto J: Redox signaling. Mol Cell Biochem 234-235: 49-62, 2002.

15. Storz P: Reactive oxygen species in tumor progression. Front Biosci 10: 1881-1896, 2005 .

16. Wang J, Li JZ, Lu AX, Zhang KF and Li BJ: Anticancer effect of salidroside on A549 lung cancer cells through inhibition of oxidative stress and phosphor-p38 expression. Oncol Lett 7: 1159-1164, 2014.

17. Okon IS and Zou MH: Mitochondrial ROS and cancer drug resistance: Implications for therapy. Pharmacol Res 100: 170-174, 2015.

18. Budina-Kolomets A, Webster MR, Leu JI, Jennis M, Krepler C, Guerrini A, Kossenkov AV, Xu W, Karakousis G, Schuchter L, et al: HSP70 inhibition limits FAK-dependent invasion and enhances the response to melanoma treatment with BRAF inhibitors. Cancer Res 76: 2720-2730, 2016. 
19. Ciocca DR and Calderwood SK: Heat shock proteins in cancer: Diagnostic, prognostic, predictive, and treatment implications. Cell Stress Chaperones 10: 86-103, 2005.

20. Sun C, Wang Z, Zheng Q and Zhang H: Salidroside inhibits migration and invasion of human fibrosarcoma HT1080 cells Phytomedicine 19: 355-363, 2012.

21. Sutton P, Borgia JA, Bonomi P and Plate JM: Lyn, a Src family kinase, regulates activation of epidermal growth factor receptors in lung adenocarcinoma cells. Mol Cancer 12: 76, 2013.

22. Summy JM and Gallick GE: Treatment for advanced tumors: SRC reclaims center stage. Clin Cancer Res 12: 1398-1401, 2006.

23. Ablin RJ, Owen S and Jiang WG: Prostate transglutaminase (TGase-4) induces epithelial-to-mesenchymal transition in prostate cancer cells. Anticancer Res 37: 481-487, 2017.

24. Lv C, Huang Y, Liu ZX, Yu D and Bai ZM: Salidroside reduces renal cell carcinoma proliferation by inhibiting JAK2/STAT3 signaling. Cancer Biomark 17: 41-47, 2016.

25. Yan L, Lin B, Gao L, Gao S, Liu C, Wang C, Wang Y, Zhang S and Iwamori M: Lewis (y) antigen overexpression increases the expression of MMP-2 and MMP-9 and invasion of human ovarian cancer cells. Int J Mol. Sci 11: 4441-4452, 2010.

26. Rajoria S, Suriano R, George A, Shanmugam A, Schantz SP, Geliebter J and Tiwari RK: Estrogen induced metastatic modulators MMP-2 and MMP-9 are targets of 3,3'-diindolylmethane in thyroid cancer. PloS One 6: e15879, 2011.

27. Lai WW, Hsu SC, Chueh FS, Chen YY, Yang JS, Lin JP, Lien JC, Tsai $\mathrm{CH}$ and Chung JG: Quercetin inhibits migration and invasion of SAS human oral cancer cells through inhibition of $\mathrm{NF}-\kappa \mathrm{B}$ and matrix metalloproteinase-2/-9 signaling pathways. Anticancer Res 33: 1941-1950, 2013.

28. Braicu EI, Gasimli K, Richter R, Nassir M, Kümmel S, Blohmer JU, Yalcinkaya I, Chekerov R, Ignat I, Ionescu A, et al: Role of serum VEGFA, TIMP2, MMP2 and MMP9 in monitoring response to adjuvant radiochemotherapy in patients with primary cervical cancer-results of a companion protocol of the randomized NOGGO-AGO phase III clinical trial. Anticancer Res 34: 385-391, 2014.

29. Liu L, Zhang J, Yang X, Fang C, Xu H and Xi X: SALL4 as an epithelial-mesenchymal transition and drug resistance inducer through the regulation of c-Myc in endometrial cancer. PloS One 10: e0138515, 2015.

30. Weinberg RA: Mechanisms of malignant progression. Carcinogenesis 29: 1092-1095, 2008.

31. Mani SA, Guo W, Liao MJ, Eaton EN, Ayyanan A, Zhou AY, Brooks M, Reinhard F, Zhang CC, Shipitsin M, et al: The epithelial-mesenchymal transition generates cells with properties of stem cells. Cell 133: 704-715, 2008.
32. Lin SY, Chang HH, Lai YH, Lin CH, Chen MH, Chang GC, Tsai MF and Chen JJ: Digoxin suppresses tumor malignancy through inhibiting multiple Src-related signaling pathways in non-small cell lung cancer. PloS One 10: e0123305, 2015.

33. Smith AL, Iwanaga R, Drasin DJ, Micalizzi DS, Vartuli RL, Tan AC and Ford HL: The miR-106b-25 cluster targets Smad7, activates TGF- $\beta$ signaling and induces EMT and tumor initiating cell characteristics downstream of Six1 in human breast cancer. Oncogene 31: 5162-5171, 2012.

34. Liu J, Bao J, Hao J, Peng Y and Hong F: HSP70 inhibits high glucose-induced Smad3 activation and attenuates epithelial-to-mesenchymal transition of peritoneal mesothelial cells. Mol Med Rep 10: 1089-1095.2014.

35. Yang J, Zhu T, Liu X, Zhang L, Yang Y, Zhang J and Guo M: Heat shock protein 70 protects rat peritoneal mesothelial cells from advanced glycation end-products-induced epithelial-to-mesenchymal transition through mitogen activated protein kinases/extracellular signal-regulated kinases and transforming growth factor- $\beta /$ Smad pathways. Mol Med Rep 11: 4473-4481, 2015.

36. Banerjee Mustafi S, Chakraborty PK and Raha S: Modulation of Akt and ERK1/2 pathways by resveratrol in chronic myelogenous leukemia (CML) cells results in the downregulation of Hsp70. PloS One 5: e8719, 2010.

37. Fang X, Jiang Y, Feng L, Chen H, Zhen C, Ding M and Wang X: Blockade of PI3K/AKT pathway enhances sensitivity of Raji cells to chemotherapy through down-regulation of HSP70. Cancer Cell Int 13: 48, 2013.

38. Banerjee Mustafi S, Chakraborty PK, Dey RS and Raha S: Heat stress upregulates chaperone heat shock protein 70 and antioxidant manganese superoxide dismutase through reactive oxygen species (ROS), p38MAPK, and Akt. Cell Stress Chaperones 14: 579-589, 2009.

39. Diao J, Yang X, Song X, Chen S, He Y, Wang Q, Chen G, Luo C, Wu X and Zhang Y: Exosomal Hsp70 mediates immunosuppressive activity of the myeloid-derived suppressor cells via phosphorylation of Stat3. Med Oncol 32: 453, 2015.

This work is licensed under a Creative Commons Attribution-NonCommercial-NoDerivatives 4.0 International (CC BY-NC-ND 4.0) License. 